\title{
Coloring, computational complexity, and culture
}

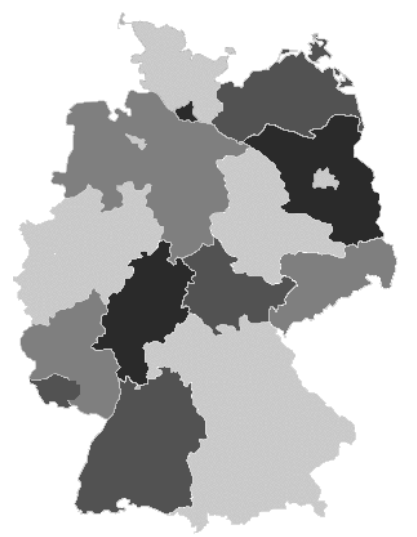
von Michael O. Albertson

In the year 2000, the Clay Mathematical Institute selected seven problems to be Millennium Prize Problems. Foremost among these is one that asks what is feasible to compute - "Is $P=N P$ ?". This paper begins with elementary ideas from graph coloring. These will be used to introduce the fundamental problem in computational complexity. Finally we explore how ideas from complexity have altered the way in which mathematicians practice their craft.

Many mathematicians believe that unsolved problems are at the core of our discipline. This view was enhanced by David Hilbert's presentation of 23 problems at the International Congress of Mathematicians in 1900. Hilbert's problems give a "high status to axiomatisation" [7]. This emphasis on the formal may have ironically led to a partial eclipse of the role of problems during much of the twentieth century. Recently problems have regained favor. Erdôs writes, "A well chosen problem can isolate an essential difficulty in a particular area, serving as a benchmark against which progress in this area can be measured" [6]. As part of our historical awareness of the importance of the Hilbert problems, many problem lists appeared during the past few years. One of these lists was noticed by the popular press.

The Clay Mathematics Institute selected seven Millennium Prize Problems [4]. Foremost among these is what Smale [11] calls computer science's gift to mathematics, "Is P = NP?". The use of foremost may be controversial, but it is at least literally true since this is the first problem listed. It is also the youngest; the most easily stated; the one with obvious, huge, practical consequences; and the one that has changed the culture of doing mathematics.

Each problem has a prize of one million dollars for its solution. Given the prizes that Erdôs offered and paid for his problems this amount seems generous [5]. Given the talent and work that is likely to be required for a solution, mathematics is once again undervalued.

This paper is an introduction to graph coloring, computational complexity, and their intricate interactions.

\section{Graph coloring}

To build a graph begin with a vertex set - imagine a (finite) set of points (say) in the plane. Within the graph some pairs of these vertices will be joined by edges - imagine straight line segments or polygonal arcs. In 1736 Euler invented graphs for his solution to the Königsburg bridge problem. It is interesting that he did not construct a geometric realization. This had to wait for the middle of the 19th century where graphs were used as models for molecules and electrical networks [3].

An $r$-coloring of a graph is an assignment of one of $r$ colors to each vertix of the graph so that if vertex $x$ is joined to vertex $y$ by an edge, then the color assigned to $x$ differs from the color assigned to $y$. Typically the colors are the integers $1,2, \ldots, r$. The left side of the figure shows a 4 -coloring of a graph $G$. The chromatic number of a graph $G$, denoted by $\chi(G)$, is the fewest number of colors that will allow a coloring. The right side shows a 3-coloring of $G$. Since $G$ contains a triangle and thus needs at least three colors, $\chi(G)=3$.

1 [Image added by editor. Source: Benedikt Großer, Stefanie Krivsky, Andreas Frommer and Katrin Schäfer, http://www. csg-germering.de/faecher/mathe/mathe/4FP/StartIn.htm] 


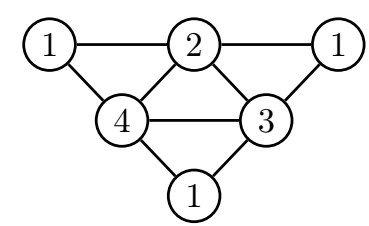

A 4-coloring of $G$

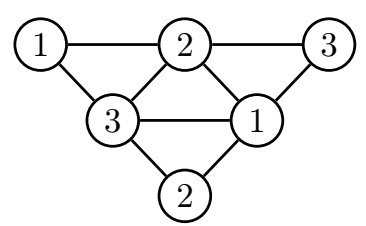

$\chi(G)=3$
Graph coloring originated with the Four Color Conjecture viz. the countries of any plane map can be colored with four colors so that any two countries that share a non-trivial boundary can be given different colors. This version can be thought of as graph coloring if we color the capitals. Perhaps this would have been a million dollar problem had it not been settled $[2,10]$.

Example 1 Many practical problems are really graph coloring in disguise.

1. In classroom scheduling problems the courses are the vertices. Two vertices are joined by an edge if the corresponding courses overlap in time. The colors are the classrooms.

2. In frequency assignment the transmitters are the vertices (e.g., cell phone towers or garage door openers). Two vertices are joined by an edge if the corresponding transmitters are sufficiently close that a signal from one would interfere with the other. The colors are the frequencies. ${ }^{2}$

3. In compiling a computer program the variables are the vertices. Two vertices are joined by an edge if the corresponding variables occur in a computation together. The colors are the fast memory locations.

In each case there is a practical advantage in minimizing the number of colors.

Let us look at the chromatic number of several families of graphs. One can realize the vertices of $P_{n}$, the path with $n$ vertices, as the integers $\{1,2, \ldots, n\}$. Here two vertices $x$ and $y$ are joined with an edge if $|x-y|=1$. We invite the reader to pick up a pencil and see that $\chi\left(P_{n}\right)=2$. One can realize $C_{n}$, the cycle with $n$ vertices, as the regular polygon with $n$ vertices. We invite the reader to prove (perhaps by induction) the following theorem.

Theorem $1 \chi\left(C_{n}\right) \leq 3$.

Since the inequality cannot be sharpened, the preceding theorem is a best possible result. Probably you have figured out the real truth, that if $n$ is even, $\chi\left(C_{n}\right)=2$ and if $n$ is odd, $\chi\left(C_{n}\right)=3$. That a best possible result could be so far from the truth is disquieting.

We generally don't talk about it, but mathematicians have insecurities about their work. We worry about correctness. Usually over time that abates. We worry about priority, "Is this new?". We consult Math. Reviews or Zentralblatt Math or we ask trusted senior colleagues. Every few years I would ask just this question of Paul Erdôs, and he would respond with either a yes or a reference. We worry if something we have done is interesting and we worry whether our work will be subsumed like Theorem 1 . We shall see that ideas from complexity help us determine that our work is important and likely to stand.

\section{Complexity classes}

We consider decision problems: questions phrased so that the answer is either yes or no.

Example 2 It is convenient to assume that all numbers that appear below are integers.

1. Is $\sqrt{s}=t$ ? We could decide this by squaring $t$.

2. Does this polynomial have a critical point at $x=$ 3 ? We could decide this by formally differentiating and plugging in.

3. Is $\operatorname{gcd}(r, s)=1$ ? Here we run the Euclidean algorithm.

4. Is this matrix invertible? We could do a Gaussian elimination.

A decision problem is said to be in $\mathrm{P}$ if there is a fast algorithm that gets the answer. Here "fast" means "requires a number of steps that is at most a polynomial in the size of the instance." Each of the decision problems above is in P. There is subtlety in the notion of the size of the instance which we shall gloss over. There are various notions of what a step consists of, but these produce the same class P. A reader interested in an excellent book on complexity should consult [8].

Some graph coloring problems are in P. For instance consider the question, given a graph $G$ is $\chi(G)=2$ ? If $G$ has no edges, then $\chi(G)=1$. If $G$ contains edges, begin by coloring any vertex 1 . Next color its neighbors 2 , their neighbors $1, \ldots$ Repeat for each component of the graph. Now if every vertex receives exactly one color, then $\chi(G)=2$. If some vertex receives two colors, then the graph necessarily contains an odd cycle and $\chi(G) \geq 3$.

In contrast consider the question, given a graph $G$ is $\chi(G)=3$ ? To provide a yes-answer one would need both a proof that $G$ is 3 -colorable and a proof that $G$ cannot be 2 -colored. One can quickly decide

2 [Editorial note: cf. the article by Eisenblätter et al., this issue, p. $18 \mathrm{ff}$.] 
if $\chi(G) \leq 2$. To test 3-colorability suppose we start coloring as in the preceding argument. If the first vertex gets color 1, without loss we can color one of its neighbors 2 . If we look at another neighbor of the first vertex its color might either be 2 or 3 . We might not know that one choice was good and the other bad until many other vertices had been colored. In short we seem to need to check an exponential (not a polynomial) number of different colorings.

What if we were good at guessing? By that we mean that as we decide which color to assign to a given vertex, we will select from those that do not immediately conflict, and that each selection we make will be consistent with a 3-coloring of the original graph, provided one exists.

A decision problem is said to be in NP (NP stands for non deterministic polynomial) if for every instance that has a yes-answer, there is a fast algorithm that will guess and verify the yes answer. One can think that the guessing requires good luck or perhaps trying all the possibilities. By the preceding paragraphs "Is $\chi(G)=3$ ?" is in NP.

There is an equivalent formulation involving the notion of a certificate that is perhaps easier to work with. A problem is in NP if for every instance that has a yes answer there is a certificate and a fast algorithm that will take the instance and the certificate and verify that the answer is yes. For the 3-coloring problem the certificate could consist of the color assignments (to show that $\chi(G) \leq 3$ ) and the vertices in an odd cycle (to show that $\chi(G) \geq 3$ ).

It is immediate that $\mathrm{P} \subseteq \mathrm{NP}$. The Millennium Problem is to determine whether the inclusion is proper.

Here are some instances of decision problems that are in NP.

Example 3 We indicate what each certificate might consist of.

1. Is $n$ prime? - Pratt discovered something like a converse to Fermat's Little Theorem [9].

2. Is $n$ composite? - Any non trivial factor.

3. Given two graphs $G$ and $H$, is $G$ isomorphic to $H$ ? - A labeling of each graph that specifies the isomorphism.

4. In a graph $G$ is there a Hamilton cycle, one that visits each vertex exactly once? - A list of the vertices in order.

5. In a finite set of positive integers are there two disjoint subsets with equal sums? - A list of the two subsets.

The problem "Is $\chi(G)=3$ ?" has the following special property. If we are interested in whether or not a given integer, say $n$ is prime, there is a transformation that takes the integer $n$ and produces a (not exponentially large) graph $G(n) . G(n)$ is constructed to have the following property:

$$
n \text { is prime } \Longleftrightarrow \chi(G(n))=3 .
$$

Thus if we had a fast algorithm to test for any graph $G$ whether $\chi(G)=3$, we could apply it to $G(n)$ and quickly decide whether $n$ is prime.

Actually much more is true. There is such a transformation for every problem in NP, i.e.,

- every instance of every problem in NP can be translated into a question about whether a particular graph is 3-colorable.

This is remarkable! Such a problem is called NPcomplete. A problem in NP can be thought of as being NP-complete if it is at least as hard as every other problem in NP. There are thousands of important NP-complete problems. The last two problems in Example 3 are NP-complete [8]. Note that problem 3 from Example 3 is thought to not be in $\mathrm{P}$, but also to not be NP-complete. Problem 1 is thought to be in $\mathrm{P}$, and therefore problem 2 as well.

If any NP-complete problem is actually in $\mathrm{P}$, then $\mathrm{P}$ $=\mathrm{NP}$. If any problem in NP is hard, then every NPcomplete problem has at best a superpolynomial algorithm to solve it, and $\mathrm{P} \neq \mathrm{NP}$. Since "Is $\chi(G)=3$ ?" is NP-complete, the question "Is $\mathrm{P}=\mathrm{NP}$ ?" is equivalent to "Is there a fast algorithm that given a graph $G$ will decide if $\chi(G)=3$ ?

Is $\mathrm{P}=\mathrm{NP}$ ? Most mathematicians and computer scientists who work on this problem suspect the answer is no. For over thirty years we have been unsuccessful at finding fast algorithms for NP-complete problems, but perhaps this only reflects a lack of imagination.

The consequences if $\mathrm{P}=\mathrm{NP}$ are enormous. There are many important problems that now seem impossible that might be doable. On the other hand public key cryptography as we currently know it might crumble. The security of cryptosystems is based on the presumed (but not proved) difficulty of certain computations.

\section{Mathematical culture}

The existence of NP-complete problems has had a profound impact on the culture of doing mathematics. If you are working on a problem that is either in or closely related to a problem in $\mathrm{P}$, then there is a risk if you don't have the complete answer. Partial results about problems in $\mathrm{P}$ are likely to be subsumed. This is what happened with Theorem 1.

There are standard ways mathematicians find work. 
- Take an open question and settle it with extra hypotheses.

- Take an open question and settle it with a weaker conclusion.

These strategies are low risk in the sense of mathematical insecurities if the original question relates to an NP-complete problem.

Let me provide a personal example.

Theorem 2 [1] Suppose $G$ is an $r$-colorable graph and $P \subseteq V(G)$ is such that the distance between any two vertices in $\mathrm{P}$ is at least 4 . Any $(r+1)$-coloring of $\mathrm{P}$ extends to an $(r+1)$-coloring of all of $G$.

The best result of this sort that one might hope for is that there are conditions that force an $r$-coloring of $\mathrm{P}$ to extend to an $r$-coloring of all of $G$. But the question, "Does an $r$-coloring of $\mathrm{P}$ extend to an $r$-coloring of $G$ ?" is NP-complete provided $r \geq 3$ [12].

If we consider what might happen if the vertices of $\mathrm{P}$ are colored with $r+1$ colors we get the question, "Does an $(r+1)$-coloring of $\mathrm{P}$ extend to an $(r+1)$ coloring of $G$ ?" It turns out that this question is also NP-complete when $r \geq 2$ [12].

That both of these questions are NP-complete leads me to believe that Theorem 2 will not be subsumed by a result that describes exactly when colorings of $\mathrm{P}$ extend to colorings of all of $G$. I am reassured.

\section{References}

[1] Michael O. Albertson, You can't paint yourself into a corner, J. Combinatorial Theory Ser B, 78 (1998), 189194.
[2] Ken Appel and Wolfgang Haken, Every planar map is four-colorable, Bull. Amer. Math. Soc. 82 (1976), 711712 .

[3] Norman L. Biggs, E. Keith Lloyd, and Robin J. Wilson, Graph Theory 1736-1936, Clarendon Press, Oxford, 1976.

[4] http://www.claymath.org/prizeproblems/

[5] Fan Chung and Ron Graham, Erdôs on graphs. His legacy of unsolved problems., AK Peters, Ltd., Wellesley, MA, 1998.

[6] Paul Erdôs, The mathematics of Paul Erdôs, I, edited by R. L. Graham and J. Nesetril, Springer-Verlag, Berlin, 1966.

[7] Ivor Grattan-Guinness, A sideways look at Hilbert's twenty-three problems of 1900, Notices American Math. Soc. 47 (2000), 752-757.

[8] Christos Papadimitriou, Computational Complexity, Addison Wesley, New York, 1994.

[9] V. R. Pratt Every prime has a succinct certificate, SIAM J. Computing 4 (1975), 214-220.

[10] Neil Robertson, Dan Sanders, Paul Seymour, and Robin Thomas, The four colour theorem, J. Combinatorial Theory, Ser. B 70 (1997), 2-44. [Editorial note: cf. http: //www.math.gatech.edu/ thomas/FC/fourcolor.html]

[11] Steven Smale, Mathematical problems for the next century, Mathematical Intelligencer, 20 No. 2 (1998), 7-15.

[12] Zsolt Tuza, Graph colorings with local constraints - a survey, Discuss. Math. Graph Theory 17 (1997), 161228 .

\section{Adresse des Autors}

Prof. Dr. Michael O. Albertson

Department of Mathematics

Smith College

Northampton, MA 01063, USA

albertson@math. smith.edu 